12

\title{
Оптимизация масс-сепараторов
}

\author{
(C) В.В. Лукашевич \\ НИЦ „Курчатовский Институт“ - ПИЯФ им. Б.П. Константинова, \\ 188300 Гатчина, Ленинградская обл., Россия \\ e-mail: lukashevich_vv@pnpi.nrcki.ru
}

Поступило в Редакцию 23 марта 2021 г.

В окончательной редакции 14 июня 2021 г.

Принято к публикации 16 июня 2021 г.

Оптимизация масс-сепараторов основана на реализации линейности исследуемой системы и следствий из теоремы Лиувилля. Рассмотрены свойства нескольких масс-сепараторов с энергией ионов $30 \mathrm{keV}$ и эмиттансом пучка, равным $4 \mathrm{~mm} \cdot \mathrm{mrad}$. Фокусировка обеспечивалась безаберрационной линзой и магнитным корректором. Фазовые диаграммы вдоль трассы пучка имели вид параллелограммов, что указывает на отсутствие геометрических аберраций. Для каждого из сепараторов разрешающая способность, вычисленная в линейном приближении, совпадала с результатами моделирования. Показано, что масс-сепаратор на базе магнита с углом поворота $54.7^{\circ}$ имел разрешающую способность около 5000 , а сепаратор на основе двух магнитов с углами поворота $45^{\circ}$ и $90^{\circ}$ соответственно имел разрешающую способность $14000-15000$.

Ключевые слова: ионная оптика, эмиттанс, линзы, фазовая диаграмма, аберрации.

DOI: 10.21883/JTF.2021.11.51540.76-21

\section{Введение}

C момента появления (1967 г.) первой ISOLDE (isotope separator on line device) системы в ЦЕРНЕ масс-сепараторы появились во многих лабораториях мира. Хотя свойства этих установок в целом и свойства их составляющих фокусирующих элементов хорошо изучены, имеющиеся масс-сепараторы не отличаются высокой разрешающей способностью. Декларированные высокие разрешающие способности сепараторов на стадии их расчета и проектирования при аппаратной реализации оказывались в несколько раз меньше расчетных. Причина заключается в том, что оптические элементы формирующих пучки ионов на входе в анализирующий магнит имеют значительные аберрации, которые нельзя проконтролировать имеющимися средствами диагностики и которые не устраняются при использовании мультипольных корректоров. Для большинства сепараторов величина эмиттанса пучка ионов неизвестна, также неизвестен и вид фазовых диаграмм по трассе пучка.

B настоящей работе при оптимизации параметров масс-сепараторов реализуются два утверждения. Во-первых, оптическая система, к которой можно отнести масс-сепараторы, может быть высококачественной только в случае, если эта система линейна. Во-вторых, согласно фундаментальной теореме Лиувилля о сохранении фазового объема, при постоянном эмиттансе пучка ионов ширина пучка тем меньше, чем больше угловой разброс траекторий частиц. В приложении к сепараторам это означает, что для увеличения разрешающей способности пучок на входе в магнит нужно стремиться сделать как можно шире. При этом, поскольку секторный магнит является фокусирующим элементом, на выходе из магнита будут увеличиваться углы наклона траекторий и соответственно уменьшаться ширина пучка в фокальной плоскости, т. е. увеличиваться разрешающая способность.

Оптимальные характеристики масс-сепаратора достигаются посредством компьютерного моделирования, заключающегося в вычислении распределения электростатических полей между электродами линзовой системы и магнитного поля магнита, решения уравнения движения, позволяющего в любом сечении пучка определить параметры траекторий частиц в пучке ионов. Эти параметры представляются в виде фазовых диаграмм, дающих возможность оценить качество фокусировки и оптимизировать фокусирующую систему. Задача заключается в достижении линейности преобразования параметров траектории. Подобная процедура описана в статьях $[1,2]$ и настоящая работа является их продолжением. В работе [1] развит метод фазовых диаграмм, на основе которого в [2] разработаны безаберрационная линза и электростатические корректоры аберраций линз и дипольных магнитов.

Измерение фазовых диаграмм путем моделирования представляется единственно возможным способом диагностики пучка частиц в силу невозможности инструментального измерения фазовых диаграмм в области электромагнитных полей. Измерения же фазовых диаграмм в свободных от полей областях пространства, например в фокальной плоскости магнита, не позволяют судить о месте появления аберраций, их величине и характере.

\section{1. Ионный источник}

Определим параметры ионного источника и величину эмиттанса пучка так, чтобы масс-сепаратор с аксиально 


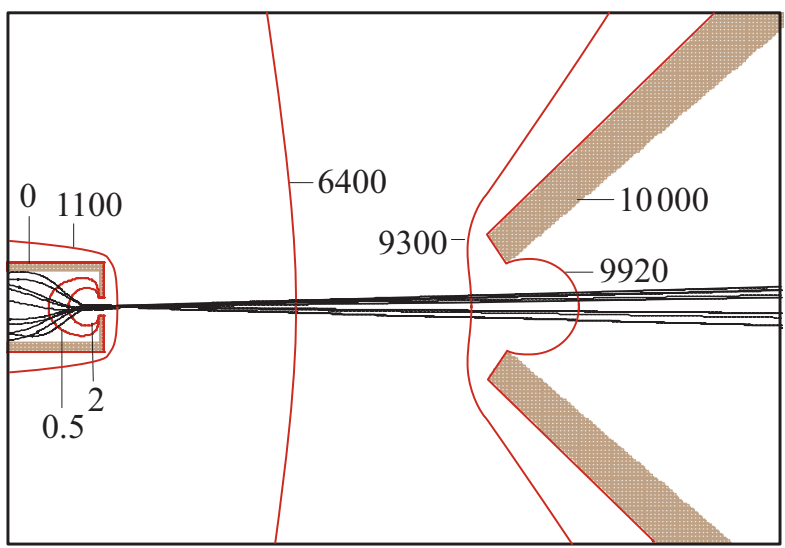

Рис. 1. Ионный источник и вытягивающий электрод. Представлены несколько эквипотенциальных линий с ионно-оптическими потенциалами в вольтах.

симметричной линзой с апертурой $100 \mathrm{~mm}$ и углом поворота $54.7^{\circ}$ имел типичную разрешающую способность порядка 1000. Этот сепаратор используется для калибровки ионного источника.

Ионный источник поверхностной ионизации в оn line масс-сепараторах представляет собой трубку, один конец которой соединен с мишенью, а на торце другого конца имеется небольшое отверстие для выхода ионов. Ионы вытягиваются и ускоряются электрическим полем, возникающим из-за разности потенциалов между источником и вытягивающим электродом. Величина ионного тока зависит от этого напряжения и тока, нагревающего трубку. С ростом вытягивающего напряжения электрическое поле глубже проникает внутрь источника, увеличивая область захвата ионов, а повышение температуры трубки увеличивает эффективность ионизации.

Из опыта эксплуатации масс-сепараторов известно, что в интервале ионных токов пикоамперы-наноамперы форма массовой линии в фокальной плоскости сепаратора не изменяется. Это свидетельствует о том, что распределение потенциалов, создаваемое вытягивающим электродом внутри источника, не искажается объемным зарядом ионов и эмиттанс пучка постоянен.

В настоящей работе описывается простая модель, в которой программа моделирования описывает рождение ионов внутри трубки источника со средней энергией $0.1 \mathrm{eV}$, что соответствует температуре в $2000^{\circ} \mathrm{K}$. Затем решается уравнение движения иона в электрическом поле, найденном при решении уравнения Лапласа.

На рис. 1 изображен моделируемый ионный источник. Торцевое отверстие имеет диаметр $1 \mathrm{~mm}$, а внутренний диаметр трубки равен $4 \mathrm{~mm}$. Расстояние между источником и вытягивающим электродом составляет $20 \mathrm{~mm}$. Внутри трубки ионы рождаются со случайными наклонами траекторий в пределах конуса с угловым раствором $40^{\circ}$ и вытягиваются электрическим полем, создаваемым вытягивающим электродом с потенциалом $10 \mathrm{keV}$. На расстоянии $0.8 \mathrm{~mm}$ от торца источника нахо- дится кроссовер пучка. Электростатический потенциал в этой точке равен $1.58 \mathrm{kV}$.

Фазовая диаграмма пучка в точке кроссовера на выходе из источника представлена на рис. 2. Границы фазовой диаграммы линейны, что в соответствии с результатами работы [1] указывает на отсутствие аберраций. Эмиттанс пучка $\eta$ равен $16 \mathrm{~mm} \cdot \operatorname{mrad}$ и при ускорении ионов до энергии $30 \mathrm{keV}$ становится равным $4 \mathrm{~mm} \cdot \mathrm{mrad}$.

Форма фазовой диаграммы зависит от начальных условий. При других значениях вытягивающего потенциала и других углах раствора конуса фазовая диаграмма может принимать вид параллелограмма.

Масс-сепаратор с таким эмиттансом пучка имеет разрешающую способность порядка 1000. Нелинейная фазовая диаграмма пучка перед магнитом представлена на рис. 3, а диаграммы в фокальной плоскости магнита и спектр двух масс $A=100$ и 100.2 этого сепаратора представлены на рис. 4.

Ионный пучок с энергией $30 \mathrm{keV}$ и эмиттансом $4 \mathrm{~mm} \cdot \operatorname{mrad}$ будет использоваться для тестирования описываемых ниже сепараторов.

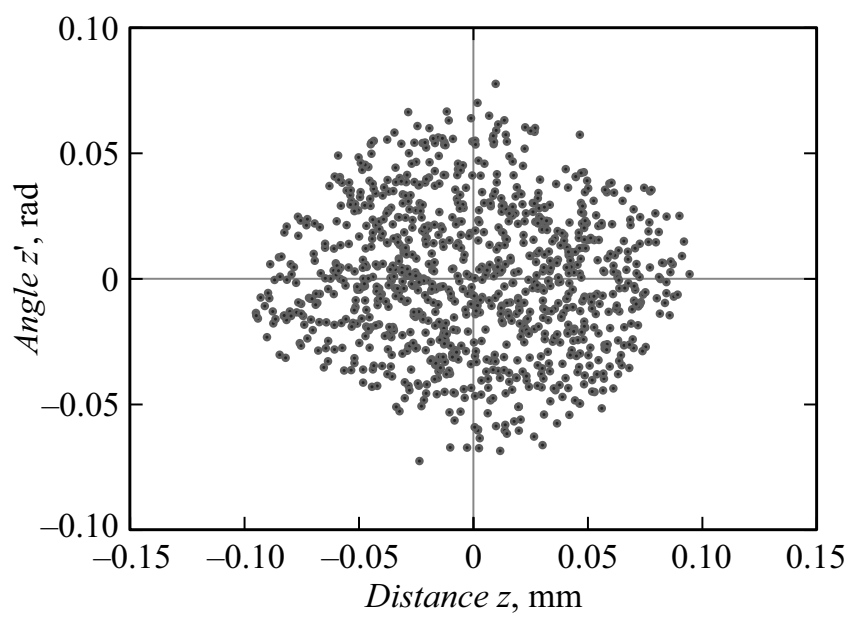

Pис. 2. Фазовая диаграмма пучка вблизи ионного источника.

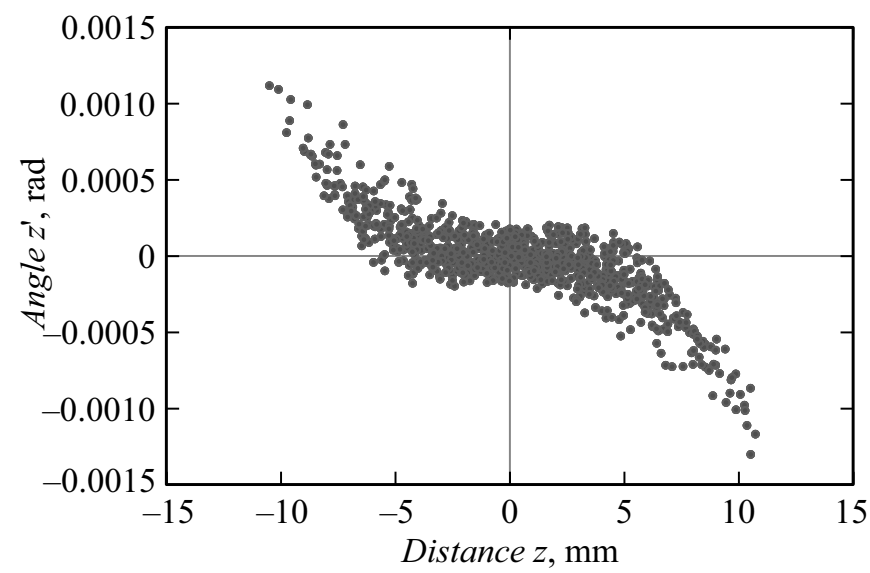

Рис. 3. Фазовая диаграмма пучка ионов перед магнитом. 

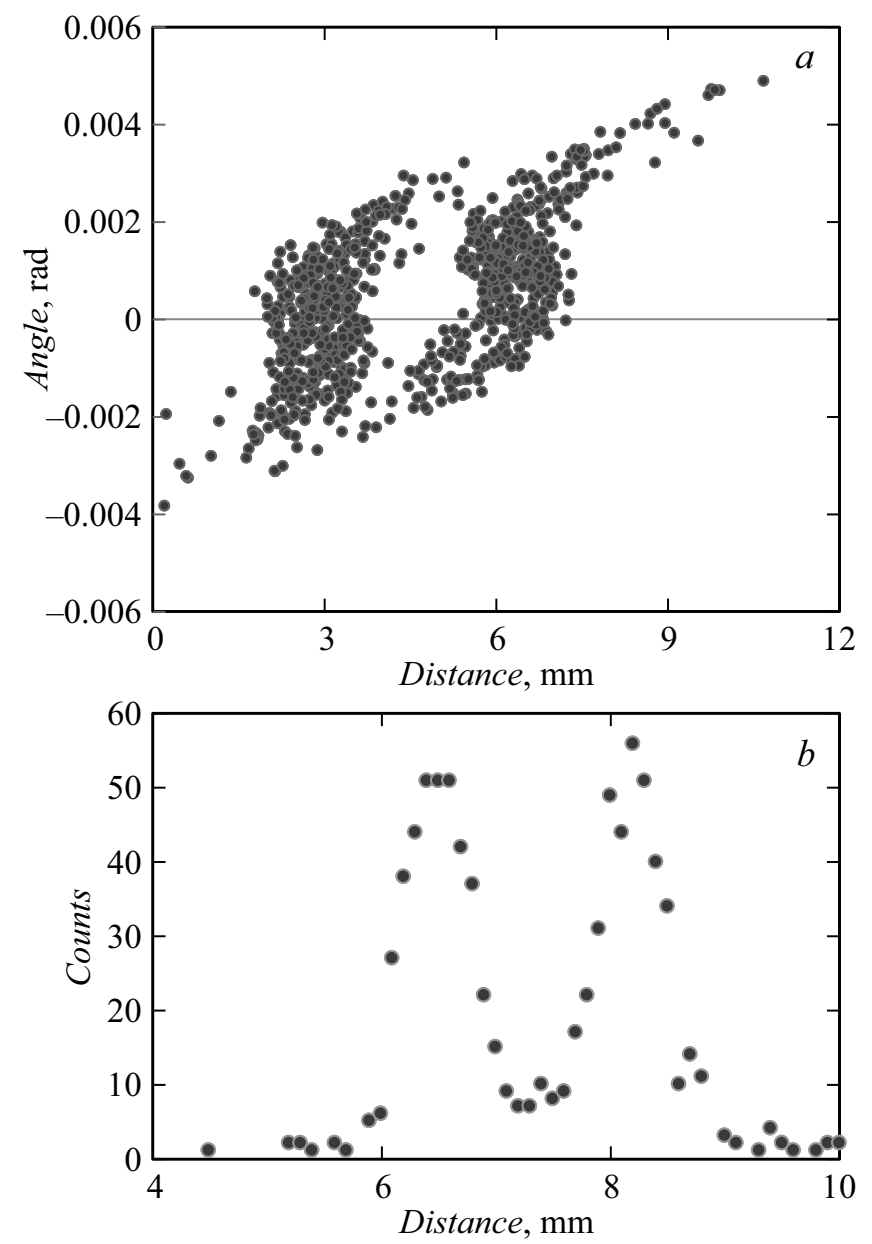

Pис. 4. $a-$ фазовые диаграммы; $b-$ спектр масс 100 и 100.2 .

В монографии [3] обсуждается вопрос, что будет с геометрическими аберрациями при уменьшении размеров оптического элемента? Будут ли они стремиться к нулю или к конечному пределу? Мнения по этому вопросу разделились, хотя доказательств того или иного утверждений не существует.

Можно указать на эквивалентное устройство, в центре которого пучок имеет такую же фазовую диаграмму, как на рис. 2. Это устройство представляет собой трубку, заполненную траекториями частиц. Траектории с нулевыми наклонами распределены по диаметру трубки $d$ и этот диаметр равен интервалу $0.2 \mathrm{~mm}$ на рис. 2. Из этого же рисунка следует, что траектории в центре трубки имеют максимальные наклоны, равные $z^{\prime}=d / l=0.08 \mathrm{rad}$. Отсюда длина трубки $l$ равна $0.2 / 0.08=2.5 \mathrm{~mm}$. Трубка с диаметром $0.2 \mathrm{~mm}$ и длиной $2.5 \mathrm{~mm}$ является миниатюрным устройством. Подобная аналогия указывает на то, что оптика ионного источника может рассматриваться как оптика малоразмерного устройства и что такое устройство не имеет аберраций. Это не доказательство, но косвенный довод в пользу того, что при уменьшении размеров электростатической оптики аберрации значительно ослабляются и, возможно, полностью исчезают.

\section{2. Классификация магнитов масс-сепараторов}

В линейном приближении уравнения движения частиц в магнитном поле в горизонтальной и вертикальной плоскостях разделяются и становятся независимыми. Решения этих уравнений для траекторий могут быть представлены в матричном виде. Эти матрицы известны с 1953 г. [4], но поскольку в явном виде они почти не встречаются в современной литературе по ионной оптике, приведем их еще раз. Это выражения (1) и (2a).

$$
\begin{gathered}
M_{z}=\left(\begin{array}{ccc}
\frac{\cos \left(\vartheta-\varepsilon_{1}\right)}{\cos \varepsilon_{1}} & \rho \sin \vartheta & \rho(1-\cos \vartheta) / 2 \\
-\frac{\sin \Omega}{\rho \cos \varepsilon_{1} \cos \varepsilon_{2}} & \frac{\cos \left(\vartheta-\varepsilon_{2}\right)}{\cos \varepsilon_{2}} & \left(\sin \vartheta+\operatorname{tg} \varepsilon_{2} \times\right. \\
0 & 0 & 1(1-\cos \vartheta) / 2
\end{array}\right), \\
M_{y}=\left(\begin{array}{cc}
1-\vartheta \operatorname{tg} \varepsilon_{1} & \rho \vartheta \\
-\frac{1}{\rho}\left(\operatorname{tg} \varepsilon_{1}-\operatorname{tg} \varepsilon_{2}\left(1-\vartheta \operatorname{tg} \varepsilon_{1}\right)\right) & 1-\vartheta \operatorname{tg} \varepsilon_{2}
\end{array}\right) .
\end{gathered}
$$

Матрица (1) преобразует столбец

$$
\left(\begin{array}{c}
z \\
z^{\prime} \\
\Delta m / m
\end{array}\right),
$$

состоящий из отклонения частицы от оси $z$, угла наклона $z^{\prime}$ и относительного разброса масс. Столбец, который преобразует матрица (2a), включает величину отклонения у и угол наклона $y^{\prime}$.

В матрицах (1), (2a) $\rho$ - радиус кривизны траектории, а угол поворота в магнитном поле $\vartheta=\Omega+\varepsilon_{1}+\varepsilon_{2}$ складывается из угла сектора $\Omega$ и угла входа пучка частиц в магнит $\varepsilon_{1}$ и угла выхода $\varepsilon_{2}$ соответственно.

С помощью этих матриц можно спроектировать магнит масс-сепаратора, руководствуясь условиями физической задачи, имеющимся доступным пространством для размещения установки и личными предпочтениями.

В общем виде секторные магниты можно классифицировать по виду пучка ионов на входе в магнит, а именно пучок приблизительно параллелен и пучок расходится.

\section{1. Секторный магнит с входным параллельным пучком}

Сначала рассмотрим магнит с нормально падающим параллельным входным пучком. Матрицы сепаратора в этом случае имеют следующий вид:

$$
S_{i}=\left(l_{i}\right)\left(M_{i}\right),
$$

где $l_{i}$ - матрицы свободных от полей промежутков между выходной гранью магнита и его фокальной плоскостью, а $i=z, y$ указывает на горизонтальную и 
вертикальную плоскости соответственно. Эти матрицы имеют простой вид $\left(l_{i}\right)=\left(\begin{array}{cc}1 & l_{i} \\ 0 & 1\end{array}\right)$.

Матричный элемент $S_{i 11}=0$ дает равенство для нахождения длин свободных промежутков $l_{z}$ и $l_{y}$, а параметры магнита вычисляются из решения системы двух уравнений:

$$
\Delta=l_{z}-l_{y}=0 \text { и } d \Delta / d \varepsilon_{2}=0,
$$

поскольку в равенстве $\frac{d \Delta}{d m}=\frac{d \Delta}{d \vartheta} \frac{d \vartheta}{d m}=\frac{d \Delta}{d \varepsilon_{2}} \frac{d \varepsilon_{2}}{d m}=\frac{d \Delta}{d \varepsilon_{2}}=0$ производная от выходного угла по массе является угловой дисперсией, которая отлична от нуля.

Первое в (4) уравнение есть условие двойной фокусировки, а второе - сохранение этого условия в широком интервале масс.

Решение системы (4) описывает известный [5] магнит с параметрами $\vartheta=54.7^{\circ}, \Omega=19.2^{\circ}$.

Для линейно сфокусированного пучка частиц фазовая диаграмма имеют вид параллелограмма, площадь которого (эмиттанс $\eta$ ) равна произведению длины параллелограмма, которую обозначим как $\bar{z}$, на высоту, представляющую полный угловой разброс $\bar{z}^{\prime}, \eta=\overline{z z}^{\prime}$.

Подставим в (2b) вместо угла ширину углового распределения $\bar{z}^{\prime}$, тогда матрица $S_{z}$ трансформирует эту величину в ширину распределения отклонения частиц, равную $S_{z 12} \bar{z}^{\prime}$. Разброс масс также дает распределение по координате $z$, равное $S_{z 13} \frac{\Delta m}{m}$. Приравняем эти величины и найдем разрешающую способность

$$
\tau=\frac{\Delta m}{m}=\frac{S_{z 13}}{S_{z 12} \bar{z}^{\prime}}=\frac{S_{z 13}}{S_{z 12}} \frac{\bar{z}}{\eta}=0.408 \frac{\bar{z}}{\eta},
$$

числовой коэффициент в этом выражении получается при подстановке угловых параметров магнита в матричные элементы.

В выражении (1) имеется четыре угловых параметpa, а независимыми являются только три. Их можно выбрать произвольным образом, например, угол падания $\varepsilon_{1}$, угол поворота частицы $\vartheta$ и угол сектора $\Omega$. Для нахождения этих параметров к системе двух уравнений (4) добавляется третье уравнение, определяющее максимум разрешающей способности в зависимости от угла $\varepsilon_{1}$. Это уравнение имеет следующий вид:

$$
\frac{d \tau}{d \varepsilon_{1}}=0
$$

Найденные таким образом параметры $\varepsilon_{1}=18.6^{\circ}$, $\vartheta=70^{\circ}$ и $\Omega=31.5^{\circ}$ имеют магнит сепаратора общего назначения (GPS) [6] в CERN. Разрешающая способность в этом случае равна

$$
\tau=\frac{m}{\Delta m}=\frac{S_{13}}{S_{12}} \frac{\bar{z}}{\eta}=0.571 \frac{\bar{z}}{\eta}
$$

Из сравнения этого выражения с соотношением (5) следует, что выигрыш в разрешающей способности составляет 1.4 раза.
В тех случаях, когда геометрия пространства не позволяет установить сепаратор на базе одного магнита, может оказаться возможным использовать конфигурацию двух магнитов, поворачивающих частицы в противоположных направлениях. В этом случае между магнитами имеется промежуточный фокус, после которого расходящийся пучок падает на второй магнит.

\section{2. Сепаратор с входным расходящимся пучком}

Источником ионов в таком сепараторе может быть промежуточный фокус или щель, на которую фокусируется пучок. Для простоты будем считать, что в вертикальной плоскости пучок параллелен. Такая ситуация имеет место при фокусировке параллельного пучка секторным магнитом с нормальным входом и выходом.

Вычислим параметры второго магнита в этом случае.

Используя выражения (1), (2a), для матриц сепаратора можем написать соответствующие выражения для движения в горизонтальной и вертикальной плоскостях:

$$
\begin{gathered}
S_{z}=l_{z 2} M_{z} l_{z 1}, \\
S_{y}=l_{y 2} M_{y} .
\end{gathered}
$$

Здесь индекс 1 указывает на расстояние от точки фокуса пучка (или щели) до передней грани магнита, а индекс 2 - на выходное расстояние от магнита до его фокальной плоскости. Поскольку ширина линии не должна зависеть от углов наклона траекторий расходящегося пучка, матричный элемент $S_{z 12}=0$. Это условие оптического изображения (или фокусировки из точки в точку), которое в единицах радиуса кривизны связывает величины $l_{z 1}$ и $l_{z 2}$ :

$$
l_{z 1}=\frac{l_{z 2} \cos \Omega / \cos \varepsilon_{2}+\sin \vartheta}{l_{z 2} \cos \Omega / \cos \varepsilon_{2}-\cos \vartheta} .
$$

Далее минимизируем размер сепаратора, положив, $\frac{d L}{d l_{22}}=0$, где $L-$ суммарная длина первого и второго промежутков. В результате получается квадратное уравнение, решение которого имеет следующий вид:

$$
\begin{gathered}
l_{z 2}=\cos \varepsilon_{2} \frac{\cos \vartheta \pm 1}{\sin \Omega}, \\
l_{z 1}=\frac{\cos \Omega+\cos \varepsilon_{2}}{\sin \Omega} .
\end{gathered}
$$

Угловые параметры магнита вычисляются так же, как и в предыдущем случае, т.е. из решения системы двух уравнений. Первое уравнение представляет собой условие двойной фокусировки, а второе - это же условие, но только для широкого интервала масс. В явном виде эта система имеет следующий вид:

$$
\begin{gathered}
\frac{1+\cos \left(\Omega+\varepsilon_{2}\right)}{\sin \Omega}-\frac{1}{\sin \varepsilon_{2}}=0, \\
\operatorname{tg} \Omega=\operatorname{tg}^{3} \varepsilon_{2} .
\end{gathered}
$$


Решением является $\Omega=\varepsilon_{2}=45^{\circ}, \vartheta=90^{\circ}$, а для свободных промежутков в единицах радиуса кривизны получаются следующие величины: $l_{z 1} / 2=l_{z 2}=l_{y 2}=\rho$, а дисперсия по массе равна $1.5 \rho$.

Разрешающая способность в линейном приближении, при котором фазовая диаграмма пучка в источнике линейна, определяется в этом случае следующим образом: $\tau=\frac{m}{\Delta m}=-\frac{S_{z 13}}{S_{z 11} \bar{z}}=\frac{S_{z 13} \bar{z}^{\prime}}{\eta}=\frac{1.5 \rho \bar{z}^{\prime}}{\eta}$, матричный элемент 11 в этом случае равен единице.

В этом выражении отношение эмиттанса к ширине распределения углов наклона равно ширине пучка в промежуточном фокусе или ширине входной щели.

Отметим, что в мировой литературе сепараторы подобного типа не описаны.

\section{3. Оптимизация масс-сепараторов}

Рассмотрим свойства сепаратора с линейной фокусирующей системой на базе секторного магнита с углом поворота $\vartheta=54.7^{\circ}$.

Устройство первого оптимизируемого сепаратора приведено на рис. 5. Перед полюсами магнита установлен короткозамкнутый экран, исключающий действие поля рассеяния магнита на ход траекторий в фокусирующей системе. Формирование пучка ионов осуществляется с помощью безаберрационой линзы и корректора аберраций магнита, описание которых дано в работе [2].

В каждой точке трассы пучка фазовая диаграмма линейна. В пределах первого, второго и третьего электродов линзы пучок расходится. Фокусировка происходит на последнем полевом промежутке и после четвертого электрода пучок становится примерно параллельным. Фазовая диаграмма пучка на выходе ионов из источника приведена на рис. 2.

Линейные свойства пучка в центре второго электрода демонстрирует рис. 6.

Фазовая диаграмма на выходе линзы (рис.7) в конце четвертого электрода перед магнитным корректором характеризует высокую степень линейности фокусировки. Максимальные углы расходимости траекторий равны $\pm 5 \cdot 10^{-5} \mathrm{rad}$.

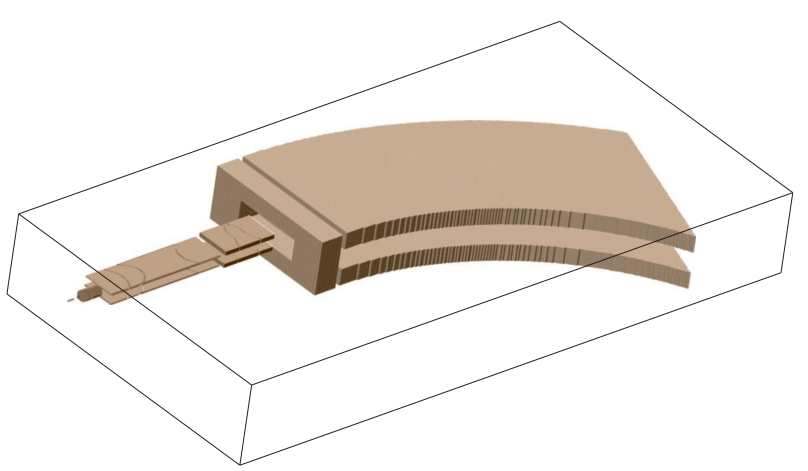

Рис. 5. Масс-сепаратор с источником ионов, безаберрационной линзой и корректором аберраций магнита.
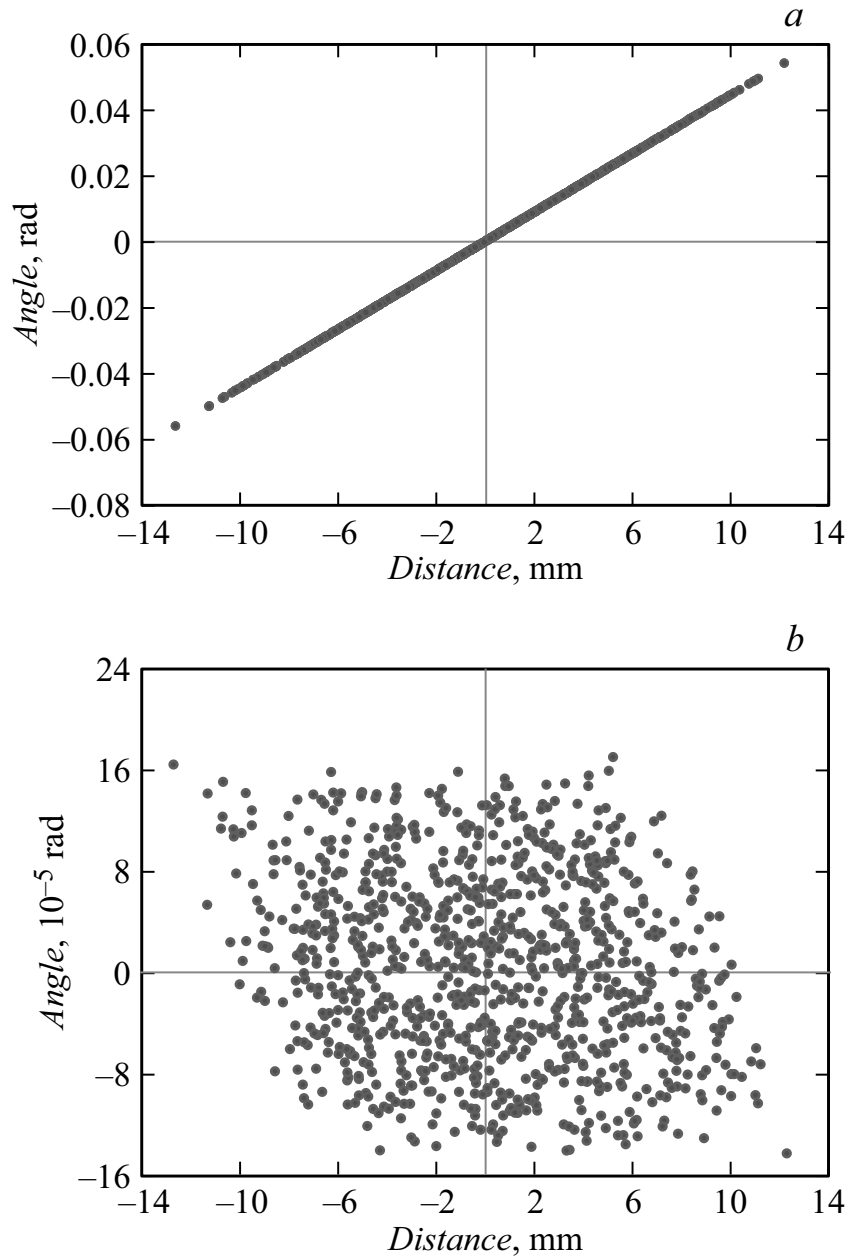

Рис. 6. $a$ - диаграмма расходящегося пучка в центре второго электрода линзы; $b-$ эта же диаграмма после приведения к виду в кроссовере.

Как описано в [2], линейность фокусировки вдоль трассы пучка достигалась с помощью итерационной процедуры, заключающейся в подборе геометрической формы электродов и электрических потенциалов на них.

Из каждой фазовой диаграммы можно получить профиль пучка частиц и распределение частиц по углам наклона траекторий. В первом случае выполняется свертка диаграммы по углу, а во втором - свертка по расстоянию до оси. Такие свертки диаграммы, изображенной на рис. 7, представлены на рис. 8 .

Подставляя в выражение (5) полную угловую ширину пучка из рис. 7 (0.12 mrad), получим ожидаемую разрешающую способность сепаратора на уровне 3000 . Если же использовать угловую ширину на половине высоты распределения рис. $8, b$, то разрешающая способность станет равной 4500. Эти числа сравним с результатами, полученными в фокальной плоскости магнита при моделировании.

Корректор с потенциалом среднего электрода $5 \mathrm{~V}$ исправляет незначительные аберрации магнита, в результате чего фазовые диаграммы двух масс $A=100$ и 


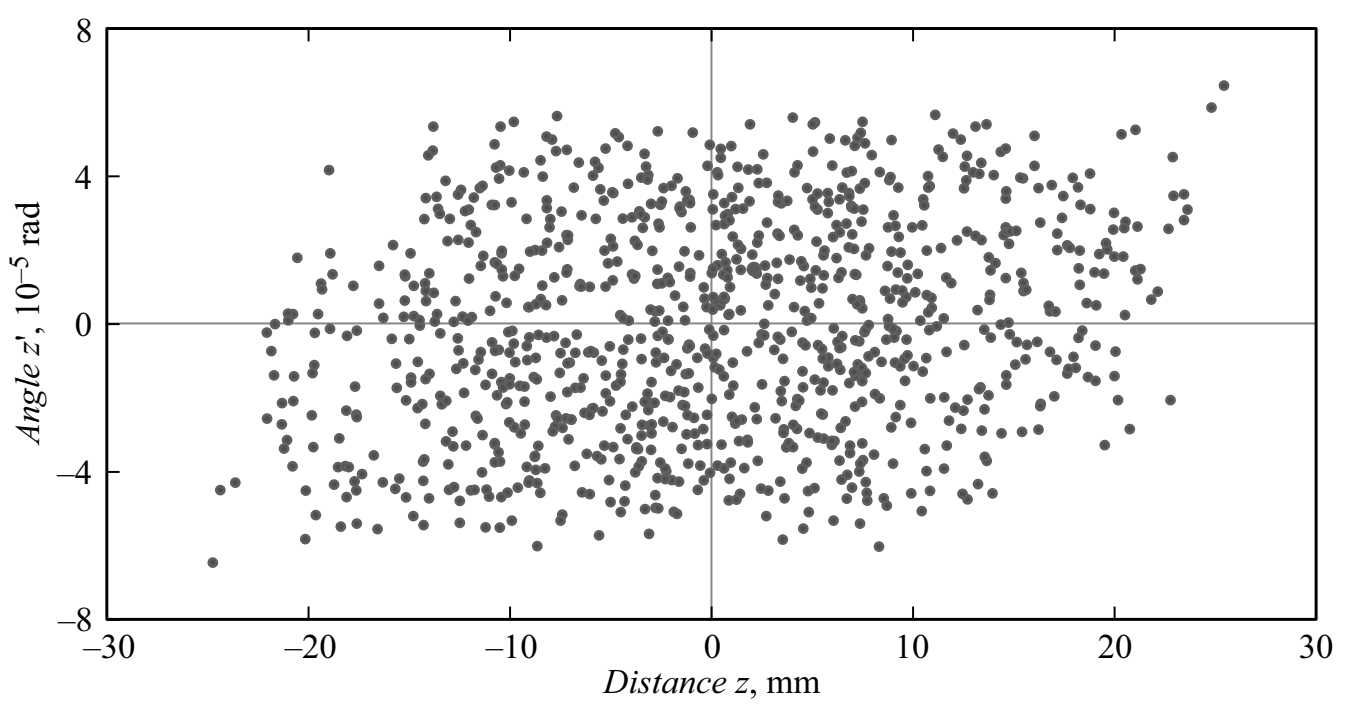

Рис. 7. Фазовая диаграмма пучка перед магнитным корректором.
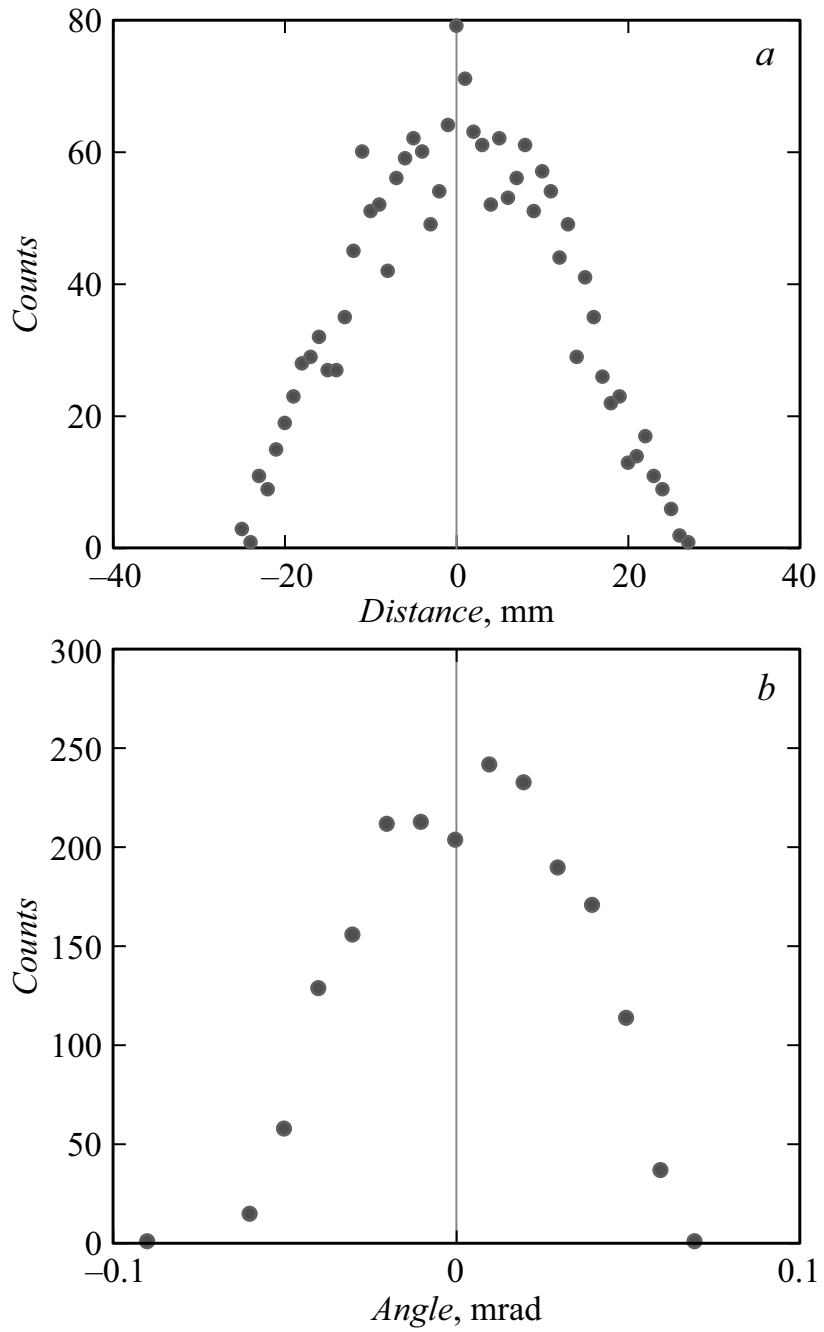

Рис. 8. Свертка фазовой диаграммы рис. 7: $a-$ по углу; $b-$ по расстоянию.
100.1 в фокальной плоскости магнита имеют линейные границы. Эти диаграммы и их свертка по углу представлены на рис. 9. Разрешающая способность, определяемая по ширине линии на половине высоты рис. 9, $b$, равна 4500-5000.

Эта разрешающая способность до 5 раз выше разрешающей способности сепараторов данного типа, имеющихся в различных лабораториях мира.

При наклонном падении пучка на переднюю грань магнита в соответствии с выражением (6) разрешающая способность будет в 1.4 раза выше.

Заметим, что полученная разрешающая способность является предельной для описанной системы и обусловлена только разбросом фазовых параметров пучка.

Моделирование влияния хроматических аберраций на разрешающую способность сепаратора приводит к выводу, что нестабильность ускоряющего напряжения порядка $10^{-5}$ и разброс энергий в ионном источнике от 0.05 до $0.2 \mathrm{eV}$ не существенны для описываемого сепаратора. Указанные аберрации вносят большой вклад только при разрешающей способности порядка 100000 и выше.

\section{1. Сепаратор на базе двух магнитов}

Рассмотрим свойства сепаратора, состоящего из секторного магнита с углом поворота частиц на $\varphi=45^{\circ}$ и только что описанного магнита с поворотом на $\vartheta=90^{\circ}$. Такое сочетание магнитов позволяет разместить их на нейтронном канале реактора ПИК Петербургского института ядерной физики без перекрытия экспериментальных зон соседних каналов.

Вид сепаратора изображен на рис. 10. Перед первым магнитом установлен ионный источник, линейно фокусирующая линза и магнитный корректор. Такой же корректор расположен перед вторым магнитом. 

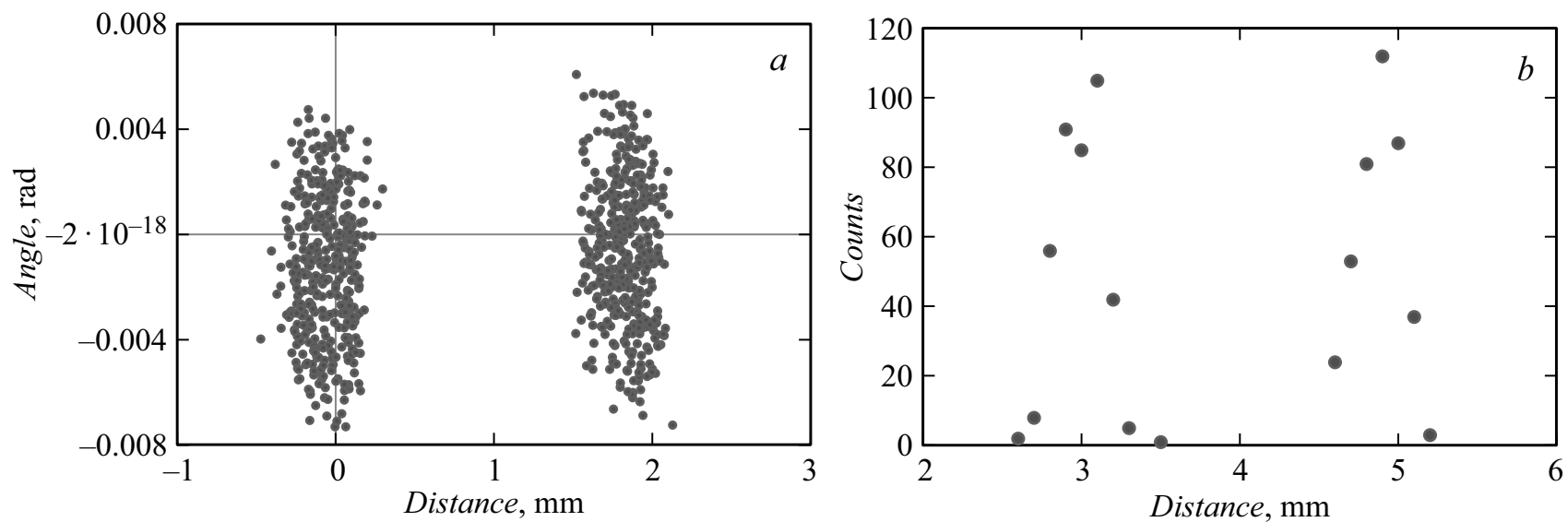

Рис. 9. Фазовые диаграммы (a) и массовые линии $(b)$ пучка ионов с массами 100 и 100.1.

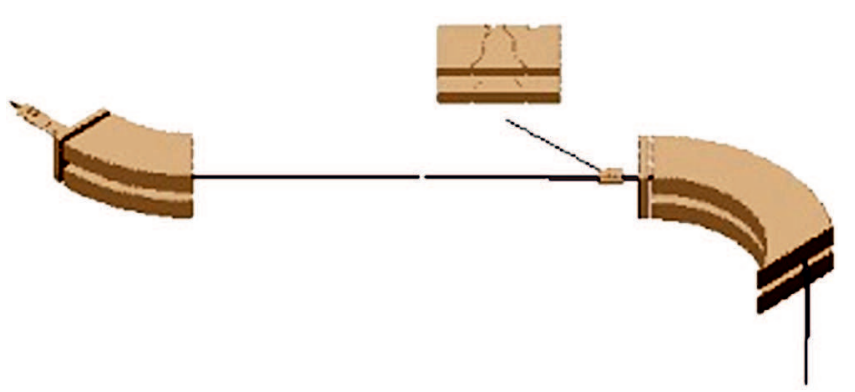

Рис. 10. Сепаратор на базе двух магнитов. На выноске изображен корректор.

Сепаратор с двумя магнитами имеет ряд полезных свойств. Во-первых, увеличивается разрешающая способность, поскольку дисперсии двух магнитов суммируются, и, во-вторых, на первом магните проще организовать защиту от нейтронов при установке сепаратора на нейтронном канале реактора.

Матрицы сепаратора получаются из матриц (7) после умножения справа на произведение $l_{z 2}^{\prime} M_{45}$, где сомножителями являются матрицы магнита и свободного промежутка магнит-фокальная плоскость. Для данного магнита этот промежуток равен радиусу кривизны.

Разрешающая способность определяется матричными элементами, имеющими следующий вид:

$$
\begin{gathered}
S_{13}=\frac{\rho}{2}\left[2-\cos \vartheta+\frac{\sin \vartheta\left(\cos \Omega+\cos \varepsilon_{2}\right)}{\sin \Omega}\right], \\
S_{12}=\frac{\rho}{\sin \varphi} .
\end{gathered}
$$

Подставив в эти соотношения вычисленные значения угловых параметров, получим величину разрешающей способности сепаратора в линейном приближении

$$
\tau=\frac{m}{\Delta m}=\frac{S_{z 13}}{S_{z 12} \bar{z}^{\prime}}=\frac{S_{z 13} \bar{z}}{S_{z 12} \eta}=\frac{1.401 \bar{z}}{\eta} .
$$

Без включенного корректора второй магнит вносит существенную нелинейность, что демонстрирует вид фазовых диаграмм двух пучков в фокальной плоскости (рис. 11).

Но при подаче потенциала $5 \mathrm{~V}$ на первый корректор и $45 \mathrm{~V}$ на средний электрод второго корректора линейность полностью восстанавливается (рис. 12) и в соответствии с рис. $12, b$ разрешающая способность, определяемая по ширине линии на половине высоты, достигает значения 14000-15000.

Поскольку система линейна, эта же величина разрешающей способности следует и из формулы (11) при подстановке ширины углового распределения на половине высоты из рис. $7, b$. Таким образом, эти результаты полностью согласованны.

Сравним свойства данного сепаратора со свойствами сепаратора высокого разрешения (HRS) в ЦЕРНЕ. Этот сепаратор на базе двух магнитов, поворачивающих частицы в противоположных направлениях, реализован на установке ISOLDE 4 [7], где первый магнит поворачивает ионы на $90^{\circ}$, а второй - на $60^{\circ}$. Сепаратор имеет сложную фокусирующую систему, состоящую из девяти квадрупольных линз, двух октупольных и одного секступольного корректоров. Кроме этого, дополнительные мультипольные корректоры установлены на входных гранях обоих магнитов, а входные и выходные

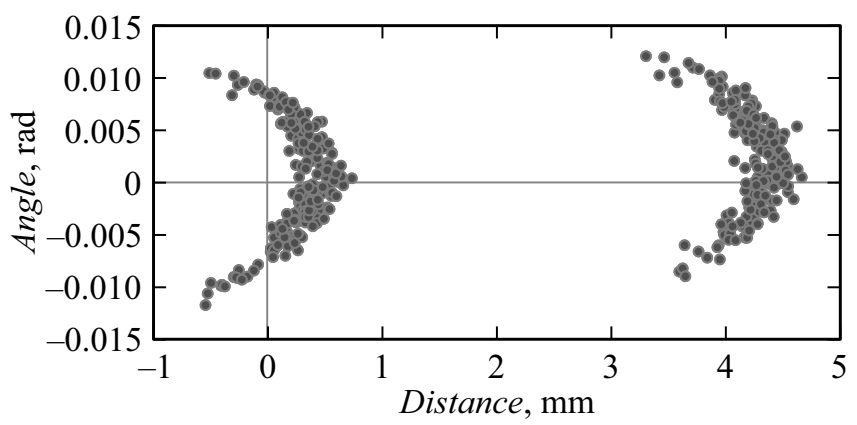

Рис. 11. Фазовые диаграммы пучков с массами 100 и 100.1 без магнитного корректора. 

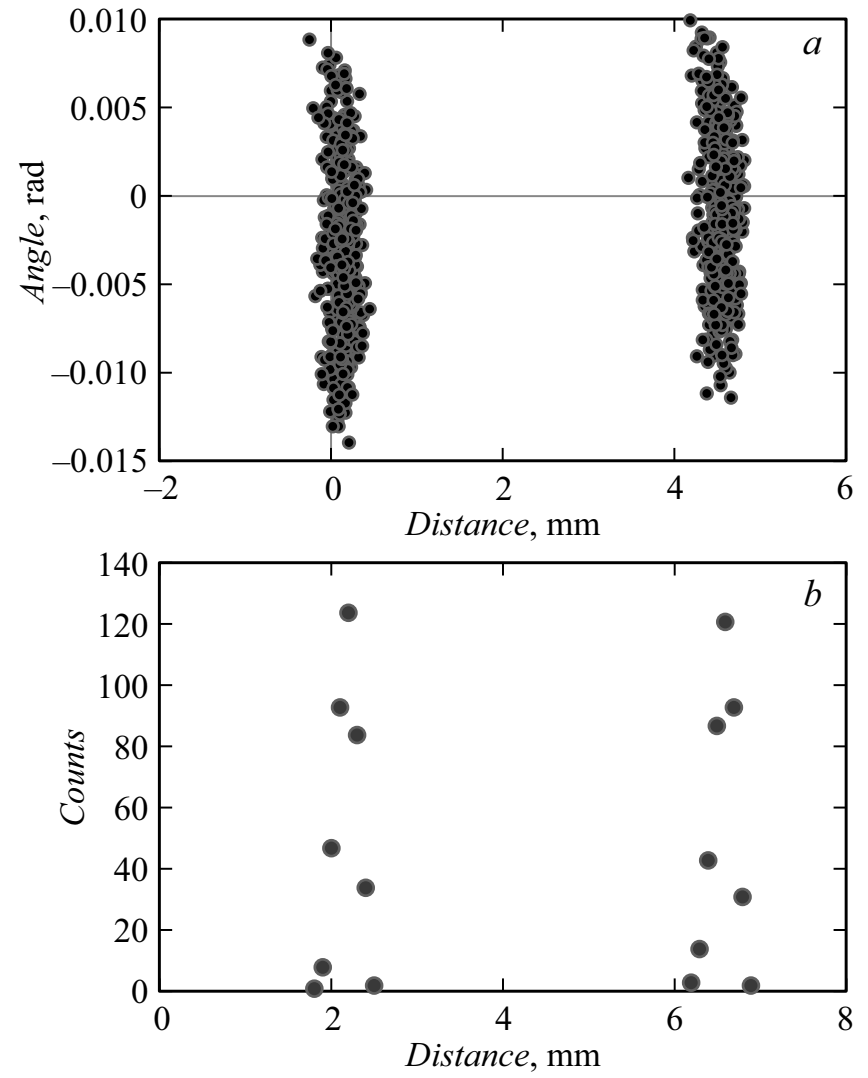

Pис. 12. $a-$ фазовые диаграммы пучков с массами $A=100$ и $100.1 ; b-$ линии этих же масс в фокальной плоскости магнита при включенном корректоре.

грани полюсов второго магнита слегка искривлены для компенсации аберраций второго порядка. При этом разрешающая способность этого сепаратора невысока и находится в интервале 3000-4500, что указывает на явную нелинейность системы.

\section{Заключение}

В работе показано, что оптимизация, основанная на требовании линейности системы и использовании следствий из теоремы Лиувилля, дает хорошие результаты. Также показано, что результаты, полученные для рассмотренных сепараторов в линейном приближении, полностью согласованы с результатами моделирования.

В заключение сделаем несколько замечаний по виду фазовых диаграмм при электростатической фокусировке и величине эмиттанса пучка, полученного с источником поверхностной ионизацией. Для линейно сфокусированных пучков фазовые диаграммы в точках кроссовера имеют вид параллелограммов, что демонстрируют рис. 2, 6, 7, 9 и 10,a. Нелинейные диаграммы представлены на рисунках 3, 4, $a$ и 11.

Вид указанных диаграмм невозможно описать с помощью эллипса с параметрами Твисса, хотя во многих работах по масс-сепараторам это представление часто используется. Например, в работе [8] приводятся результаты измерения эмиттанса пучка с энергией $30 \mathrm{keV}$ для сепаратора с источником с поверхностной ионизацией и аксиальными линзами. Измерительное устройство помещалось за фокальной плоскостью магнита с углом поворота ионов $54.7^{\circ}$. В результате измерений для эмиттанса определен интервал $\pi \cdot(10-20) \mathrm{mm} \cdot \operatorname{mrad}$. Цифры в скобках являются произведением длин полуосей эллипса. Этот же интервал используется в анализе [7]. Скорее всего, эти пределы завышены на порядок. Если взять среднее значение интервала, то эмиттанс будет равен $\pi \cdot 15=45 \mathrm{~mm} \cdot \mathrm{mrad}$. Тогда для получения разрешения в 14000 в соответствии с линейной формулой (12) ширина линейно сфокусированного пучка должна быть равна 450 и $500 \mathrm{~mm}$ в соответствии с формулой (5) для сепаратора, изображенного на рис. 5. Это нереально большие величины, значительно превышающие апертуру используемых линз.

Для повышения разрешающей способности рассмотренных сепараторов необходимо сконструировать новую линейно фокусирующую линзу с увеличенными размерами, что позволит при том же эмиттансе получить более широкий пучок.

\section{Финансирование работы}

Работа выполнена в ПИЯФ в рамках служебного задания.

\section{Конфликт интересов}

Автор заявляет, что у него нет конфликта интересов.

\section{Список литературы}

[1] В.В. Лукашевич. ЖТФ, 90 (3), 471 (2020). DOI: $10.21883 / J T F .2020 .03 .48934 .90-19$

[2] В.В. Лукашевич. ЖТФ, 90 (6), 1016 (2020). DOI: $10.21883 /$ JTF.2020.06.49292.288-19

[3] М. Силадьи. Электронная и ионная оптика (Мир, М.,1990)

[4] H. Ewald, H. Hintenberger. Methoden und Anwendungen der Massenspectroskopie (Verlag Chemie, Weinheim, 1953)

[5] G. Rudstam. Nucl. Instr. Meth., 139, 239 (1976).

[6] E. Kugler. Hyperfine Interactions, 129, 23 (2000).

[7] T.J. Giles, R. Gatherall, V. Fedoseev, U. Georg, E. Kugler, J. Lettry, M. Lindroos. Nucl. Instr. Meth. B, 204, 497 (2003); CERN/PS 2002-057(OP).

[8] F. Wenander, J. Lettry, M. Lindroos. CERN/PS 2002-045(PP). 